\title{
Performance Comparison of Fresh and Spray \& Wait Protocol through ONE Simulator
}

\author{
Puneet Garg ${ }^{1}$, Ashutosh Dixit ${ }^{2}$, Preeti Sethi ${ }^{3}$ \\ ${ }^{1}$ Research Scholar, J. C. Bose University of Science \& Technology YMCA, Faridabad, India \\ ${ }^{2}$ Professor, J. C. Bose University of Science \& Technology YMCA, Faridabad, India \\ ${ }^{3}$ Assistant Professor, J. C. Bose University of Science \& Technology YMCA, Faridabad, India \\ Email: ${ }^{1}$ puneetgarg.er@gmail.com, ${ }^{2}$ dixit_ashutosh@rediffmail.com, ${ }^{3}$ preetisethi22@gmail.com
}

\begin{abstract}
Opportunistic Networks (OppNets) are becoming the prime interest for researchers day-by-day due to the large scope of further research into it. An opportunistic network is used to transmit data in an environment of intermittent connectivity. OppNet offers a variety of routing protocols based on different strategies. Each protocol has some pros and cons. Among the available ones, Fresh Routing Protocol and Spray-and-wait Routing Protocol are the most efficient routing protocols in terms of performance during data transmission. This paper aims to compare these two different routing protocols through simulation on the ground of standard performance metrics. It is believed that this simulation comparison will help upcoming researchers in the selection of appropriate routing protocol as per their requirement.
\end{abstract}

Keywords: Opportunistic Networks, Routing Protocols, Fresh Routing protocol, Spray-and-wait protocol, ONE simulator

\section{Introduction}

An opportunistic network is a form of Delay Tolerant Network which is specifically designed to operate in the situation where node connectivity may or may not remain stable for a long time. According to Kaur et al [1], OppNet is considered a derivative of Mobile Ad hoc Networks (MANETs) with some updated features. Therefore, OppNets and MANETs have many similarities in their functionalities during data transmission like both have no central control of data communication and participating node/nodes themselves decide the route during the communication process. But, in some scenarios, OppNet is considered superior to MANETs like Inter-planetary communication, Jungle surveillance, Underwater communication, etc. where participating node may or may not be connected to further intermediate nodes for data transmission between two endpoints in a stable manner.

Opportunistic Networks provides the feature of Storecarry-and-forward to every other participating node, which means, node stores the message which it is supposed to forward till it gets the successful receiving from the destination node [2]. Due to this, data transmission in OppNet remains robust, secure, and safe. This feature of OppNet makes it the best choice for implementation in the environment of intermittent connectivity or high delays.

Nayyar et al [3] described some key points concerning to OppNets which are described below:

1. Node participating in OppNet requires high buffer space as it stores the message until the successful delivery of the message which increases the overall storage requirements of the network.

2. OppNet can tolerate variations in data transfer rate and the issue of frequent connection failure during data transmission.

3. OppNet requires a high amount of energy in its nodes, as the participating nodes may have to wait for a long time for connecting to another node for data transmission.

4. OppNet focuses on secure delivery than fast delivery of data.

\subsection{OppNet Applications}

OppNet offers several varieties of real-life applications, some of the key applications are mentioned below:

1. ZebraNet [4], an Opportunistic Network-based Framework developed by Princeton University to monitor Zebra in the forests of Kenya.

2. Lasso [5], an Android-based app which uses Opportunistic Networks in the background and is developed for maintaining communication via Bluetooth among a group of tourists visiting a smart city.

3. Saratoga [6], an OppNet based framework, is used to transfer sensed data taken by a sensory satellite to the base station on Earth. It is currently in use by NASA

4. Schiavone et al [7] revealed the fact that Opportunistic Networks may also be used to develop Airborne Networks.

5. Shared Wireless Info-Station Model (SWIM) [8], an opportunistic Network-based framework is used to monitor whales' behavior by the sensors tied on their back, these sensors forward their sensed data opportunistically to the nearest Base Station.

6. Menon et al [9] surveyed and concluded that Opportunistic Networks may be used to establish 
Underwater communication using acoustic waves as a communication medium.

\subsection{Outline of the paper}

Section-1 describes a general introduction and applications delivered by OppNet. Section-2 and Section-3 explain Fresh and Spray-and-Wait Routing Protocol respectively. Section-4 is dedicated towards ONE Simulator and simulation parameters used for evaluating protocols' performance in the focussed research. Section-5 shows the performance of both mentioned protocols through analysis of the comparative graphs. The paper is being concluded in Section-6.

\section{Fresh Protocol}

Ferriere et al [10] developed FResher Encounter SearcH (FRESH) Protocol. It was initially developed for Mobile Ad hoc Networks (MANETs), but, was later adapted for OppNet as well. Fresh Protocol belongs to the category of Social-relationship based routing protocols in Opportunistic Networks. Garg et al [11] surveyed through simulation and declared Fresh Protocol as the best routing protocol among all categories of routing protocols available in Opportunistic Networks.

The working of Fresh protocol is primarily based on encounter experiences between two nodes during data forwarding. It assumes that all nodes available in OppNet are in motion; therefore, two nodes may or may not remain connected for a long time. In Fresh Protocol, the sender node prefers to select intermediate node/nodes with recent encounter experience for data forwarding towards the destination node.

\section{Spray \& wait Protocol}

Spyropoulos et al [12] introduced a Spray-and-wait protocol with a prime focus to reduce the unnecessary traffic on intermediate nodes during data transmission in OppNet. Spray-and-wait protocol belongs to the category of Floodingbased protocols in OppNet. It does not require any encounter experience or timestamp of connectivity between two nodes in OppNet for data communication. Many survey papers [13] [14] [15] expressed the efficient performance of Spray-andwait protocols over other popular routing protocols in OppNet.

As the name suggests, Spray-and-wait Protocol works in two phases i.e. Spray Phase and Wait Phase. The spray phase involves forwarding data packets to a group of nodes immediately connected with the sender node with the belief that one or some of these nodes will help in forwarding data packets towards the destination node like the flooding approach. The Wait phase involves waiting by the sender node for acknowledgment of successful delivery from the destination node. If acknowledgment comes from the destination node within a pre-decided time limit, the communication process is considered successful otherwise sender node resends the same data packet to another group of nodes in contact. This process repeats until the sender node receives the successful delivery acknowledgment from the destination node.

\section{Simulation Setup}

Although there are numerous simulators available for OppNet simulation, however, as per kuppusamy et al [16], ONE (Opportunistic Network Environment) Simulator is widely used simulator globally. Therefore, this paper focuses on ONE simulator for our aimed research to make this research available for the larger research community associated with OppNet simulation.

\subsection{ONE Simulator}

The simulation work carried out in this paper is performed in ONE Simulator. ONE simulator was initially developed by Aalto University, Finland, and is freely available to use [17] [18].ONE (Opportunistic Network Environment) simulator is the most popular simulator for OppNet simulation across the globe []. Its framework has been depicted in Figure 1.

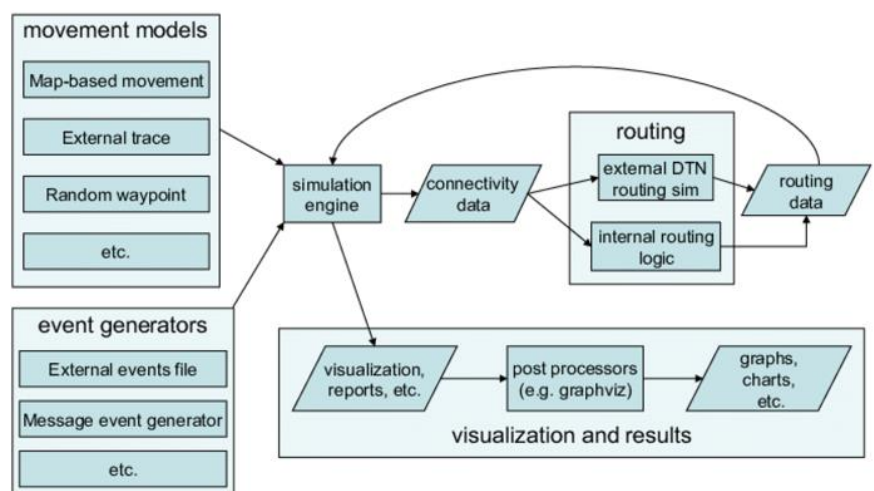

Figure 1: Framework of ONE Simulator (Source: Keränen et al [17])

ONE performs simulation using java as back-end technology. Its primary functions include modelling of routing, mobility analysis, and inter-node data transmission through discrete events. It supports a graphical user interface through an interactive and comprehensive console. It can also be handled through a keyboard interface. Outputs obtained by ONE simulation can be analyzed graphically through a plotting tool like Graphviz.

\subsection{Common Parameters}

Following are the common parameters taken constant in case of simulation experimented in this paper to get the standard and unbiased results mentioned in Table 1 below: 
Table 1: Common parameters taken for the simulation study

\begin{tabular}{ll}
\hline Parameters & Values \\
\hline Simulation Coverage & $\begin{array}{l}4000 \text { x } 3000 \mathrm{Sq} . \text { Meters } \\
\text { (12 Square KMs) }\end{array}$ \\
Simulation Time & 24 Hours \\
Movement Model & Cluster Movement \\
Time-To-Live (per Message) & 4 Hours \\
Scenario Update Interval & $0.1 \mathrm{Second}$ \\
Communication Medium & Wi-Fi (High Speed) \\
Wi-Fi Interface Speed & $1 \mathrm{Mbps}$ \\
Wi-Fi Interface Range & $15 \mathrm{Meters}$ \\
Wi-Fi Interface Interval & $32 \mathrm{Seconds}$ \\
Node movement speed & From $0.5 \mathrm{~m} / \mathrm{s}$ to $1.5 \mathrm{~m} / \mathrm{s}$ \\
Transmission Range & $11 \mathrm{Meters}$ \\
Message Size & From $200 \mathrm{~KB}$ to $500 \mathrm{~KB}$ \\
Warm-Up period & $30 \mathrm{Minutes}$ \\
Buffer Size & $15 \mathrm{MB}$ \\
\hline
\end{tabular}

\subsection{Performance Metrics}

Following six parameters are considered for inspecting the better routing protocol among the considered two:

1. Node Density: Node Density plays a vital role in determining the performance of any routing protocol. Besides, it also represents a real-life scenario such as the sparse number of nodes may represent a general scenario of a park or restaurant whereas high node density represents a scenario of a busy market or stock market place in any city. Therefore, Node Density has been taken as a prime factor to judge any protocol. In this paper, both protocols have been evaluated started from node density of 50 to the node density of 500 with an increment of 50 in each comparison.

2. Delivery Probability: It is the possibility of successful data transmission and calculated from the value 1 . The higher value of Delivery Probability denotes the good performance of a Routing Protocol.

3. Overhead Ratio: It represents the occupancy of network resources during data transmission. A protocol is considered good if it delivers the data transmission with a minimum possible Overhead ratio.

4. Average Latency: It is the average time taken by a Data to completely travel from source node to destination node via intermediate node. A protocol is considered efficient if it has minimum possible Average Latency.

5. Average Hop Count: It is the average number of intermediate nodes used during data dissemination from the source node to the destination node. Less number of Average Hop Count during data transmission is considered fast communication, but, in the case of opportunistic networks, It may or may not be true.
6. Average Buffer Time: It is the average time taken by an intermediate node to hold all messages to be transmitted during data transmission. Its less value indicates fast transmission as the node deletes the data after it gets successful acknowledgment from the destination node.

\section{Results and Analysis}

Following results have been obtained from the aimed simulation of both protocols i.e. Fresh Protocol and Sprayand-Wait Protocol:

1. Delivery Probability: As the Delivery Probability is concerned, performances of both protocols have been shown in Figure 2 below.



Figure 2: Delivery Probability versus Node Density of Fresh and Spray-and-wait Protocol

Fresh Protocol leads initially up to the node density of 280, but, gradually it seems to fall as after the density's further growth whereas Spray-and-wait protocol retains its progressive delivery probability with the growth in Node Density an average.

2. Overhead Ratio: The graph of comparative performance of both protocols has been depicted through Figure 3 below: 


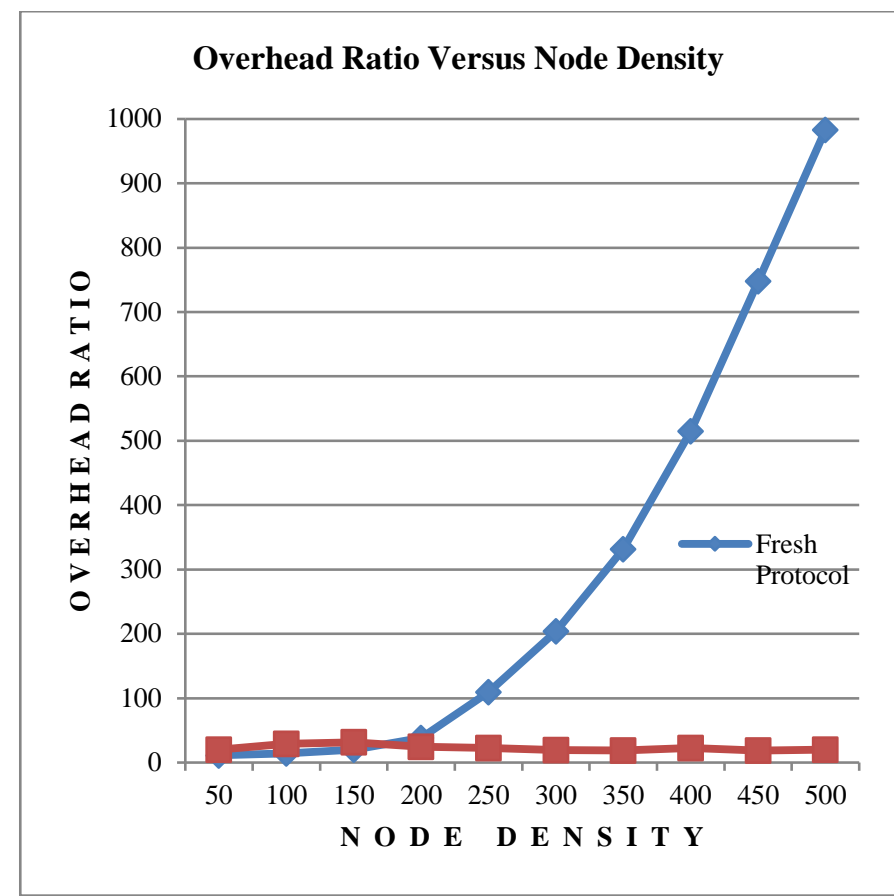

Figure 3: Overhead Ratio versus Node Density of Fresh and Spray-and-wait Protocol

The overhead ratio of both protocols remains almost bare minimum up to the node density of 200, but after that Fresh Protocol achieves continuous increasing overhead ratio with increasing Node Density whereas spray-and-wait protocol maintains a considerable low overhead ratio from the starting up to the end.

3. Average Latency: The relative performances of the two discussed protocols have been shown via Figure 4 below:

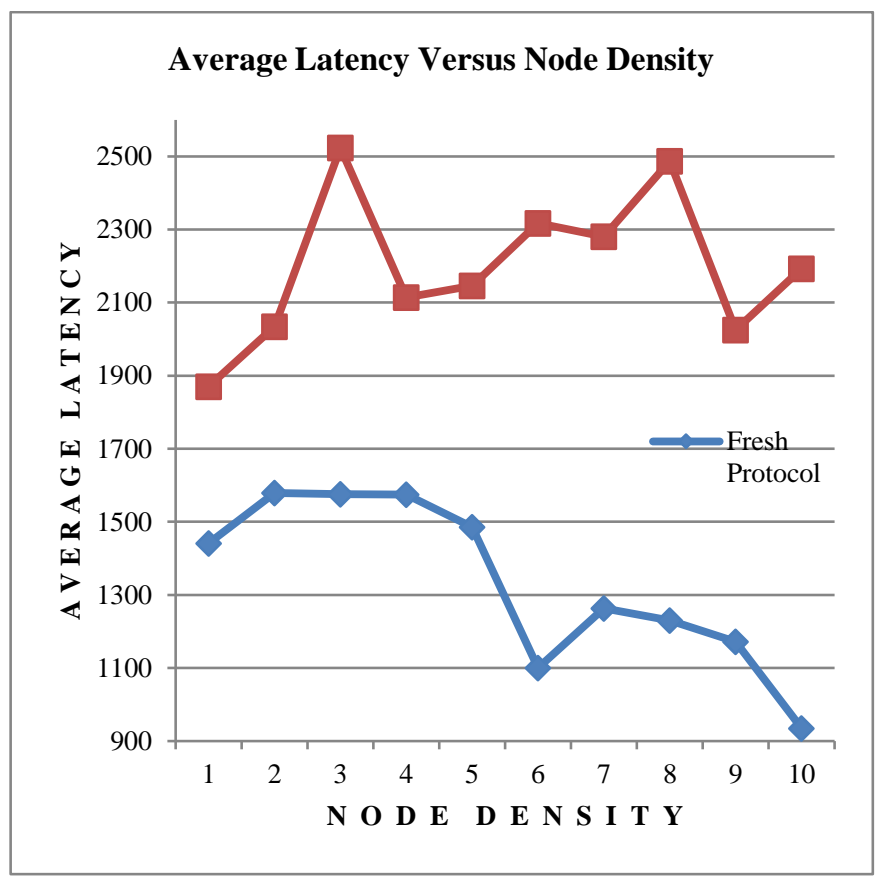

Figure 4: Average Latency versus Node Density of Fresh and Spray-and-wait Protocol
It has a very surprising thing that both protocols have an entirely different performance with every case of Node Density although both protocols are considered best among their respective category of routing protocols. Fresh Protocol shows the clear unsurpassed performance over Spray-and-wait Protocol in every case discussed over here.

4. Average Hop Count: The performance of mentioned protocols on the criteria of Average Hop Count has been shown in Figure 5 below:

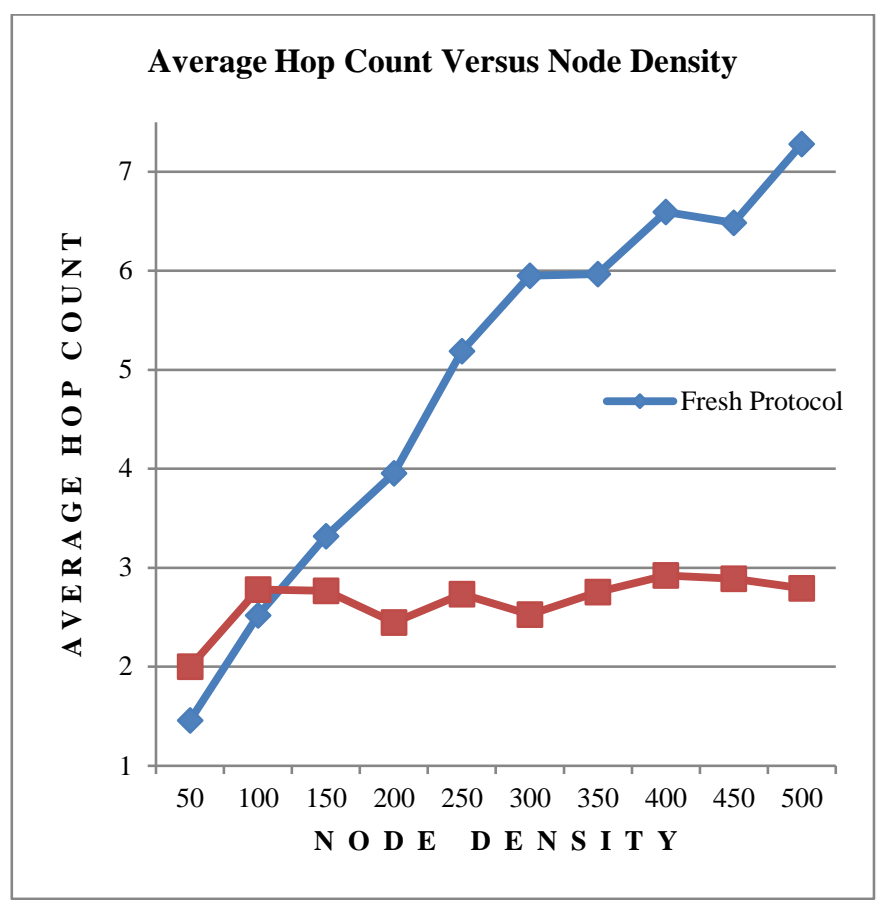

Figure 5: Average Hop Count versus Node Density of Fresh and Spray-and-wait Protocol

Fresh and Spray-and-wait protocols require almost equal Average hop Count up to the node density of 100, but after that, the requirement of hops continuously increases with the increase in node density whereas spray-and-wait protocol requirements for an average number of hops remain uniform till they starting to the end.

5. Average Buffer Time: Average Buffer Time consumption requirements of both protocols for node density has been depicted through Figure 6 below: 


\section{Average Buffer Time Versus Node Density}

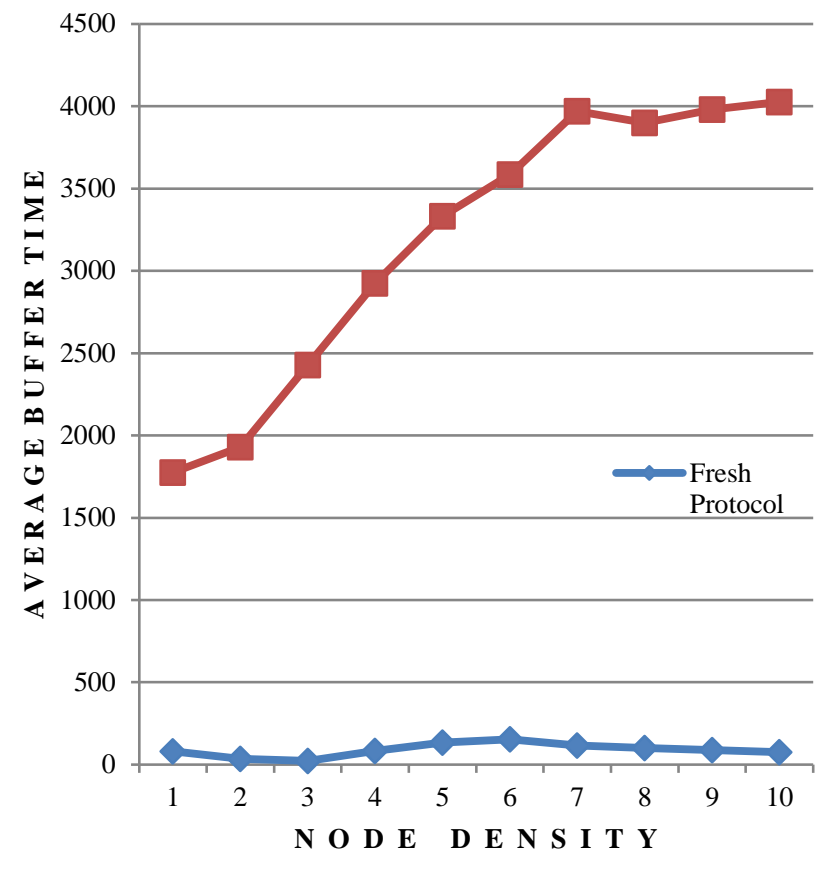

Figure 6: Average Buffer Time versus Node Density of Fresh and Spray-and-wait Protocol

Fresh Protocol requires very little time for using node buffer space on average whereas spray-and-wait protocol requirement for average buffer time goes on the increase with the growth in node density. The huge gap between the performance concerning to Average Buffer Time of both protocols may be clearly understood via Figure 6 shown above.

As per the comparison is concerned, Spray-and-wait protocol exhibits better performance than Fresh Protocol in terms of Delivery Probability, Overhead ratio, and Average number of Hops whereas Fresh Protocol has better performance over Spray-and wait Protocol on the ground of Average Latency and Average Buffer Time. The performances of both protocols proved them as nearly equal contenders. The survey conducted by Garg et al [11] reveals the fact that the Average Latency and Average Buffer Time parameters are more important than the Overhead ratio and Average number of Hops in the evaluation of protocol performance. Therefore, on that basis, Fresh Protocol must be considered as a better protocol than Spray-and-wait Protocol.

\section{Conclusion}

Opportunistic Networks were introduced along with their prime applications at the beginning of the paper. Fresh and Spray-and-wait protocols were explained thereafter. Both protocols were compared with the simulation in ONE simulator with varying numbers of nodes in the network. The behavior of both protocols was recorded and compared on the platform of six standard parameters. As per the observation, Spray-and-wait Protocol proved that it is better than Fresh Protocol and vice-versa. Based on the literature survey, the fact came to light that the parameters viz. Average Latency and Average Buffer Time play a vital role in determining the Network performance, due to which, Fresh protocol may be regarded as better than Spay-and-Wait Protocol.

\section{References}

[1] Kaur, N., \& Mathur, G. (2016). Opportunistic networks: A review. IOSR Journal of Computer Engineering (IOSR-ICE), 18(2), 20-26.

[2] Prabha, C., Kumar, S., \& Khanna, R. (2016). Analysis of routing and forwarding protocols in opportunistic networks. Procedia Computer Science, 85, 891-898.

[3] Nayyar, A., Batth, R. S., Ha, D. B., \& Sussendran, G. (2018). Opportunistic networks: present scenario-a mirror review. International Journal of Communication Networks and Information Security, 10(1), 223-241.

[4] Zhang, P., Sadler, C. M., Lyon, S. A., \& Martonosi, M. (2004, November). Hardware design experiences in ZebraNet. In Proceedings of the 2nd international conference on Embedded networked sensor systems (pp. 227-238).

[5] Saloni, M., Julien, C., Murphy, A. L., \& Picco, G. P. (2017, September). Lasso: A device-to-device group monitoring service for smart cities. In 2017 International Smart Cities Conference (ISC2) (pp. 1-6). IEEE.

[6] Wood, L., Eddy, W. M., Ivancic, W., McKim, J., \& Jackson, C. (2007, September). Saratoga: a DelayTolerant Networking convergence layer with efficient link utilization. In 2007 International Workshop on Satellite and Space Communications (pp. 168-172). IEEE.

[7] Schiavone, L. J. (2004, October). Airborne networkingapproaches and challenges. In IEEE MILCOM 2004 Military Communications Conference, 2004. (Vol. 1, pp. 404-408). IEEE.

[8] Small, T., \& Haas, Z. J. (2003, June). The shared wireless infostation model: a new ad hoc networking paradigm (or where there is a whale, there is a way) In Proceedings of the 4th ACM international symposium on Mobile ad hoc networking \& computing (pp. 233-244).

[9] Menon, V. G., \& Prathap, P. J. (2016, October). Comparative analysis of opportunistic routing protocols for underwater acoustic sensor networks. In 2016 international conference on emerging technological trends (ICETT) (pp. 1-5). IEEE.

[10] Dubois-Ferriere, H., Grossglauser, M., \& Vetterli, M. (2003, June). Age matters: efficient route discovery in mobile ad hoc networks using encounter ages. In Proceedings of the 4th ACM international symposium on Mobile ad hoc networking \& computing (pp. 257-266).

[11] Garg, P., Dixit, A., Sethi, P., \& Pinheiro, P. R. (2020). Impact of Node Density on the QoS Parameters of 
Routing Protocols in Opportunistic Networks for Smart Spaces. Mobile Information Systems, 2020.

[12] Spyropoulos, T., Psounis, K., \& Raghavendra, C. S. (2005, August). Spray and wait: an efficient routing scheme for intermittently connected mobile networks. In Proceedings of the 2005 ACM SIGCOMM workshop on Delay-tolerant networking (pp. 252-259).

[13] Juyal, V., Pandey, N., \& Saggar, R. (2016, April). Impact of varying buffer space for routing protocols in delay tolerant networks. In 2016 International Conference on Communication and Signal Processing (ICCSP) (pp. 2152-2156). IEEE.

[14] Dhurandher, S. K., Sharma, D. K., Woungang, I., \& Chao, H. C. (2011, December). Performance evaluation of various routing protocols in opportunistic networks. In 2011 IEEE GLOBECOM Workshops (GC Wkshps) (pp. 1067-1071). IEEE.

[15] Alajeely, M., Doss, R., \& Ahmad, A. A. (2018). Routing protocols in opportunistic networks-a survey. IETE Technical Review, 35(4), 369-387.

[16] Kuppusamy, V., Thanthrige, U. M., Udugama, A., \& Förster, A. (2019). Evaluating forwarding protocols in opportunistic networks: trends, advances, challenges and best practices. Future Internet, 11(5), 113.

[17] Keränen, A., Ott, J., \& Kärkkäinen, T. (2009, March). The ONE simulator for DTN protocol evaluation. In Proceedings of the 2nd international conference on simulation tools and techniques (pp. 1-10).

[18] Liu, X., \& Chen, Y. (2013). Report of A DTN Simulator-THE ONE. 